\title{
To Improve Internal RFT by Eliminating Wrapper Fouling with Bumper Issue in MF 9500 Models
}

\author{
Rajesh S, Aravindhan S, Srinivasan V
}

\begin{abstract}
Right First Time (RFT) is one of the internal business measure to ensure the quality of the product. This project deals with the elimination of wrapper fouling with bumper issue in MF 9500 Models. The wrapper is A class part which is assemble in front of tractors which when getting damage will lead to customer dissatisfaction. The wrapper damage at field results in customer / warranty claims thus increasing the external cost to the company. The wrapper damage issue is due to fouling issue in tractors and that issue is taken up for this study.. Initially PFMEA study conducted for wrapper assembly process. Further based on PFMEA results, a systematic analysis is done through cause and effect diagram to identify the other causes for wrapper damage issue. Since the cause and effect analysis not able to reveal the real root causes. Two models is proposed with modification and performance of the model is studied and compared. It is observed that proposed models are capable of reducing the wrapper damage considerably. It is also noted that modification in the proposed model reduces the operator inspection time and fatigue.
\end{abstract}

Keywords : RFT, PFMEA, wrapper fouling, wrapper damage.

\section{INTRODUCTION}

In TAFE organization we follow Total Productive Maintenance (TPM) . Based on this practice we the team implement all the improvements in the plant. Total productive maintenance (TPM) is a method of maintaining and improving the integrity of production and quality systems through the machines, equipment, employees and the supporting processes. TPM can be of great value and its target is to improve core business processes .The main Objective of TPM is

- Zero Breakdown

- Zero Accident

- Zero Defects

- Zero Waste

A. TPM PILLARS

Total productive maintenance (TPM) consists of 8 pillars.

1. Autonomous Maintenance $(\mathrm{JH})$

Revised Manuscript Received on December 5, 2019

* Correspondence Author

Rajesh S*, Department of Mechanical Engineering, Kalasalingam Academy of Research and Education, Krishnankoil, India. Email: s.rajesh@klu.ac.in

Aravindhan S, Department of Mechanical Engineering, Kalasalingam Academy of Research and Education, Krishnankoil, India. Email: aravindhantqm@gmail.com

Srinivasan V, Department of Mechanical Engineering, Kalasalingam Academy of Research and Education, Krishnankoil, India. Email: vsri@tafe.com
2. Individual Improvement (KK)

3. Planned Maintenance (PM)

4. Development Maintenance (DM)

5. Quality Maintenance (QM)

6. Education and Training (E\&T)

7. Safety, Health \& Environment (SHE)

8. Office TPM (OTPM)

\section{B. QM PILLAR}

This issue deals with Quality Maintenance (QM) Pillar. In QM pillar one of the important methodologies is Figure of 8 approach which helps to solve the problem in 7 steps. Some of the analysis is:

- PM Analysis

- ABCD Approach

- QM matrix

\section{B.1 PM Analysis}

$\mathrm{P}-\mathrm{M}$ analysis is an advanced articulation of TPM. This eight step process has been proven to reduce chronic losses to zero and has raised technological expertise in many manufacturing environments. P-M Analysis is eliminating chronic problems that have been neglected or unresolved in the past. P-M Analysis was specially developed to overcome the weaknesses of traditional methods.

$\mathrm{P}-\mathrm{M}$ analysis is more than a methodology as it is a different way of thinking about the problem and the context in which ever they occur. P- M Analysis is a systematic Problem-Solving Philosophy for Chronic Losses. The term $\mathrm{P}-\mathrm{M}$ Analysis comes from the following origin: $\mathrm{P}$ Phenomena (non), Physical; M - Mechanism, Relationship (Machine, Man/Woman, Material, and Method). It is designed to help your TPM teams analyze and eliminate chronic problems that have been neglected or unresolved in the past.

Through P-M Analysis, teams really get in touch with their equipment. Its unique skill-building process improves technological know-how while delivering solutions to persistent problems. The first four steps of this rigorous 8 -step program help teams isolate and understand the root causes of defects and failures within main equipment mechanisms and peripheral systems. The final four steps provide a systematic approach for effectively controlling those causes. 
A critical concept in P-M Analysis is physical analysis -a way of thinking about how defects and failures are generated that forces us to look at the physical principles involved and to quantify the changes in the relationship between the equipment mechanisms and product parts involved. When a proper physical analysis is carried out, teams are far less likely to overlook important factors or to waste time pursuing unrelated ones. Although not a cure-all, P-M Analysis has reduced chronic losses to zero and raised technological expertise in many manufacturing environments.

\section{PROBLEM DEFINITION}

Right First Time (RFT) trend is attached below. Losses during manufacturing of tractor due to the quality defects is high and affects cost and delivery

RFT $=\frac{\text { No of Tractors direct OK without defect }}{\text { No of Tractors produced }} \underline{\mathrm{x} 100}$
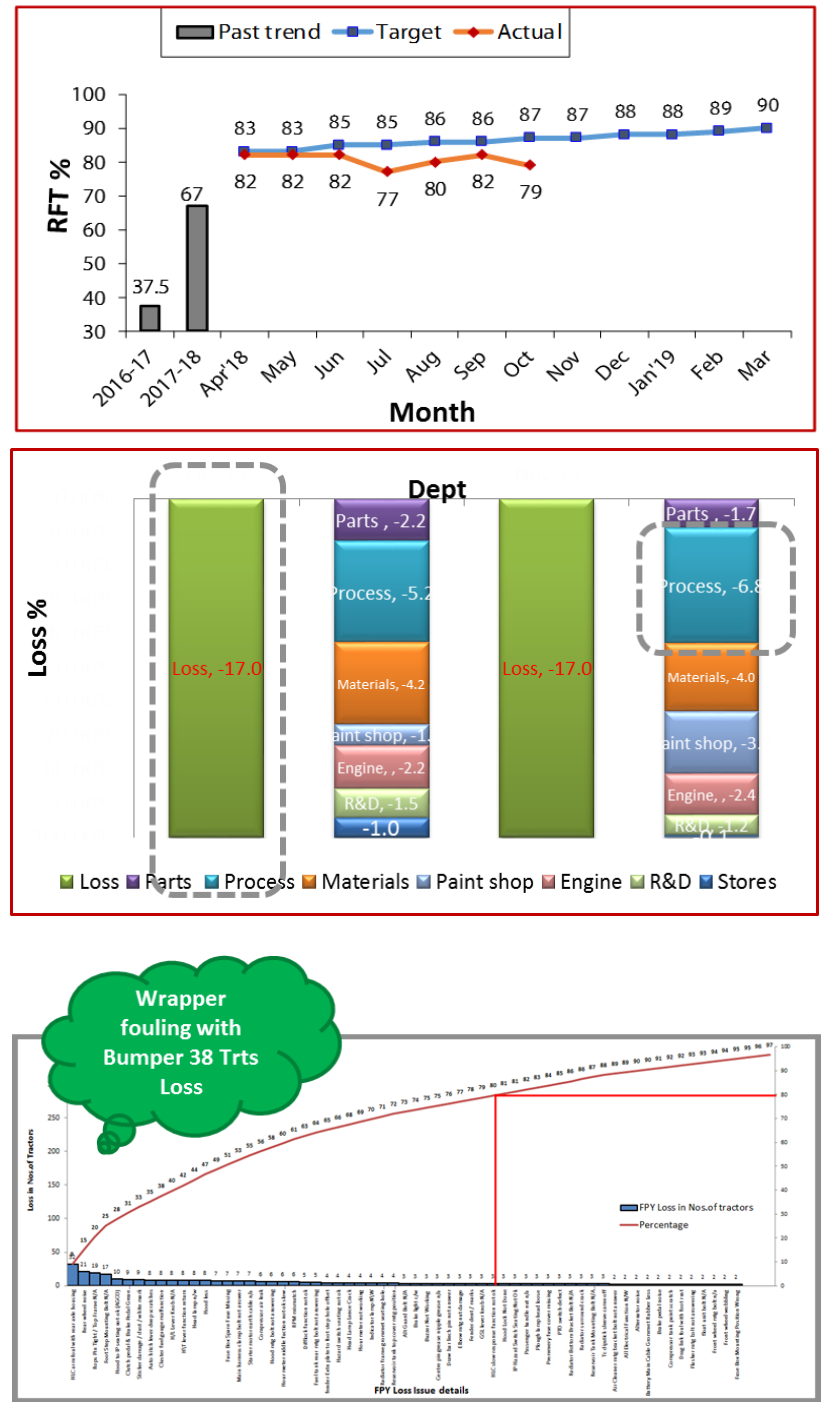

Fig. 1, $2 \&$ 3. With help of Pareto analysis the Wrapper Fouling with Bumper issue is considered as top priority and selected the same.

\section{METHODOLOGY}

STEP 1 : IDENTIFY EXISTING SITUATION

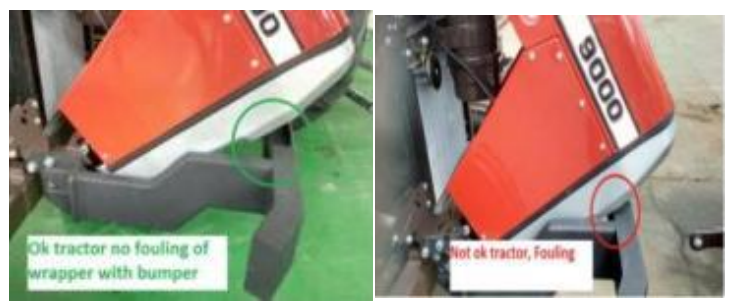

Fig. 4. Wrapper to bumper gap spec $21 \mathrm{~mm}$, actual 0 mm (After opening)

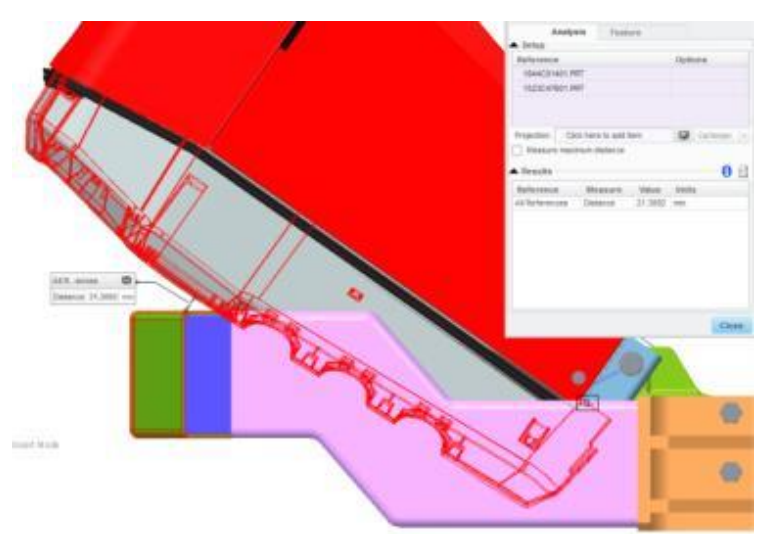

Fig. 5. Wrapper is Fouling with Bumper leading to damages after Hood swinging for multiple times.

TABLE-I: WRAPPER FRAME

\begin{tabular}{|l|l|c|c|}
\hline \multicolumn{1}{|c|}{ Function unit } & \multicolumn{1}{c|}{ Check point } & Requirement & Result \\
\hline Wrapper frame angle & To be within the Spec & $90^{\circ}$ & $90 / 91^{\circ}$ \\
\hline $\begin{array}{l}\text { Bottom hood support } \\
\text { bracket angle }\end{array}$ & To be within the Spec & $90^{\circ}$ & $90^{\circ}$ \\
\hline $\begin{array}{l}\text { FES mounting to wrapper } \\
\text { mounting distance }\end{array}$ & To be within the Spec & 140.8 & $140.4 / 140.6$ \\
\hline $\begin{array}{l}\text { Wrapper mounting } \\
\text { bracket distance }\end{array}$ & To be within the Spec & 37 & $36.88 / 37.05$ \\
\hline $\begin{array}{l}\text { Bottom hood support } \\
\text { bracket bending distance }\end{array}$ & To be within the Spec & 17 & $17.09 / 17.21$ \\
\hline $\begin{array}{l}\text { Imner width bottom hood } \\
\text { support bracket }\end{array}$ & To be within the Spec & 426 & $425.8 / 426.54$ \\
\hline
\end{tabular}

TABLE-II: WRAPPER

\begin{tabular}{|l|c|c|c|}
\hline \multicolumn{1}{|c|}{ Function unit } & \multicolumn{1}{c|}{ Check point } & Requirement & Result \\
\hline Depth Frame mounting & To be within the Spec & 74.6 & $74.4 / 74.8$ \\
\hline $\begin{array}{l}\text { Pitch distance Frame } \\
\text { mounting }\end{array}$ & To be within the Spec & 267 & $267 / 268$ \\
\hline $\begin{array}{l}\text { Pitch distance Frame } \\
\text { mounting }\end{array}$ & To be within the Spec & 236 & $236 / 237$ \\
\hline $\begin{array}{l}\text { Pitch distance Frame } \\
\text { mounting }\end{array}$ & To be within the Spec & 176 & $175 / 177$ \\
\hline $\begin{array}{l}\text { Pitch distance Frame } \\
\text { mounting }\end{array}$ & To be within the Spec & 29 & $29 / 30$ \\
\hline
\end{tabular}

TABLE-III: BUMPER

\begin{tabular}{|l|l|c|c|}
\hline \multicolumn{1}{|c|}{ Function unit } & \multicolumn{1}{|c|}{ Check point } & Requirement & Result \\
\hline $\begin{array}{l}\text { Mounting plate face to front } \\
\text { pumper face height }\end{array}$ & To be within the Spec & 5 & $4.81 / 6.30$ \\
\hline Inner width mounting plate & To be within the Spec & $452+2$ & $452 / 454$ \\
\hline $\begin{array}{l}\text { Inner width bumper cross } \\
\text { stiffner }\end{array}$ & To be within the Spec & 565 & $566 / 557$ \\
\hline $\begin{array}{l}\text { Mounting plate face to } \\
\text { mounting hole distance }\end{array}$ & To be within the Spec & 30 & $30.55 / 31.16$ \\
\hline
\end{tabular}

Published By: 


\section{TABLE-IV: FRONT ENGINE SUPPORT}

\begin{tabular}{|l|c|c|c|}
\hline \multicolumn{1}{|c|}{ Function unit } & \multicolumn{1}{|c|}{ Check point } & Requirement & Result \\
\hline Wrapper stopper angle & To be within the Spec & $55^{\circ}$ & $54^{\circ} 45^{\circ} / 55^{\circ} 32^{\prime}$ \\
\hline $\begin{array}{l}\text { Wrapper mounting to } \\
\text { machining surface distance }\end{array}$ & To be within the Spec & 25 & $24.9 / 25.7$ \\
\hline $\begin{array}{l}\text { Wrapper mounting to casting } \\
\text { surface distance }\end{array}$ & To be within the Spec & 34.2 & $33 / 33.6$ \\
\hline $\begin{array}{l}\text { Sump face to wrapper } \\
\text { mounting hole distance }\end{array}$ & To be within the Spec & 507 & $507.05 / 507.2$ \\
\hline
\end{tabular}

STEP 2 : RESTORE

If all the parts investigation is $\mathrm{OK}$ in above step, directly the step 2 is PASS.

STEP 3 : ANALYSE CAUSE

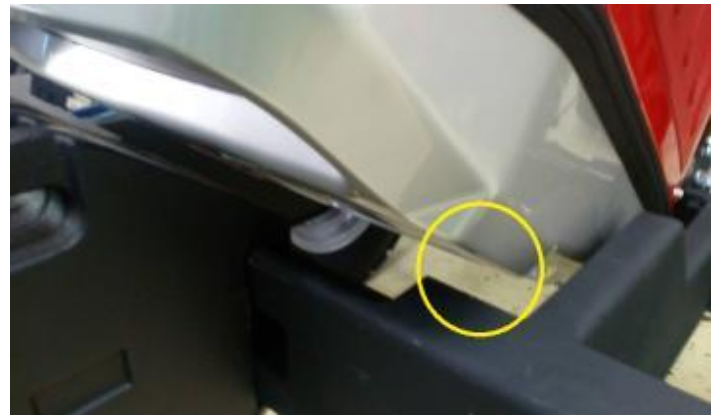

Fig. 6. Before swinging

Gap between wrapper and bumper is $21 \mathrm{~mm}$ during first swing

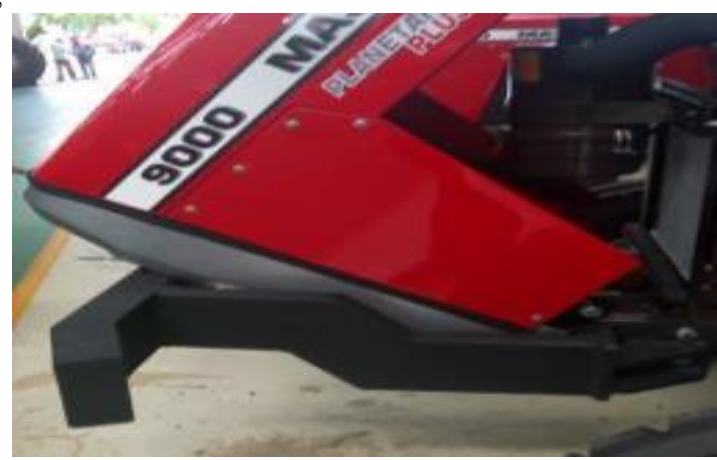

Fig. 7. After Swinging- 5 Times

TABLE-V: GAP BETWEEN WRAPPER AND BUMPER IS ONLY 7 MM AND WRAPPER IS DAMAGED

\begin{tabular}{|l|l|l|l|}
\multicolumn{2}{l}{ DAMAGED } \\
\hline Hood open and close swing & LH & RH & Remarks \\
\hline 1st time & $20 \mathrm{~mm}$ & $21 \mathrm{~mm}$ & OK \\
\hline 2nd time & $16 \mathrm{~mm}$ & $15 \mathrm{~mm}$ & OK \\
\hline 3rd time & $12 \mathrm{~mm}$ & $13 \mathrm{~mm}$ & OK \\
\hline 4th time & $10 \mathrm{~mm}$ & $9 \mathrm{~mm}$ & Wrapper is fouling with bumper \\
\hline 5th time & $8 \mathrm{~mm}$ & $7 \mathrm{~mm}$ & $\begin{array}{l}\text { Wrapper is completely damage in } \\
\text { fouling area }\end{array}$ \\
\hline
\end{tabular}

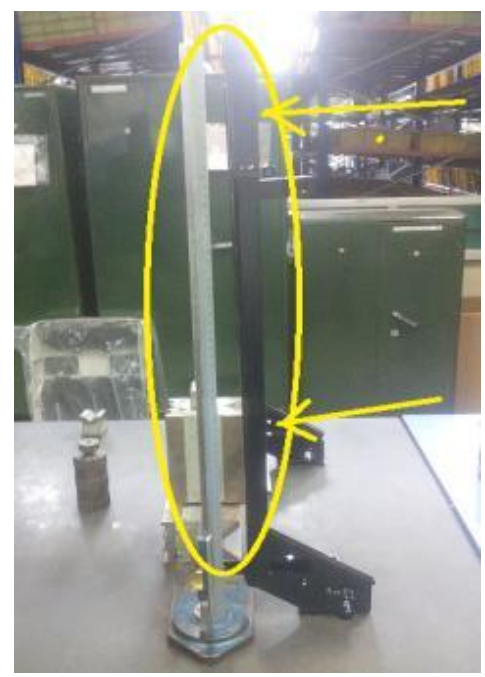

Fig. 8. Wrapper frame before fitment in tractor

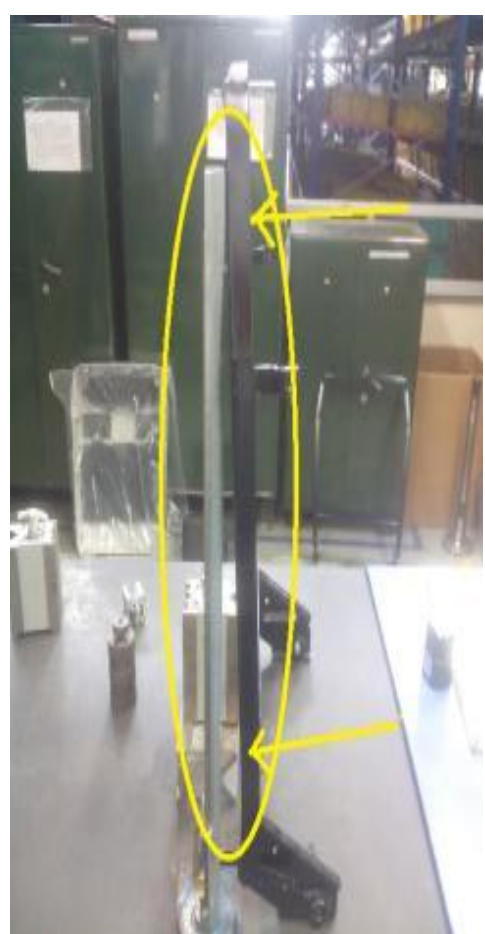

Fig. 9. Wrapper frame after assembly (dismantled from the tractor)

\begin{tabular}{|c|c|c|c|}
\hline Function unit & \multicolumn{1}{|c|}{ Check point } & Before Fitment & After assembly- hood swinging \\
\hline Wrapper frame angle & $\begin{array}{l}\text { To be within the Spec } 90 \\
\text { Deg }\end{array}$ & $90^{\circ}$ & $\begin{array}{c}94-97 \text { Deg - Wrapper frame is } \\
\text { deformed }\end{array}$ \\
\hline
\end{tabular}

Physical analysis using ABCD description - Hand Sketch

(A) Hood Assembly - Swinging

(B) Wrapper Frame

(C) Tensile Strength In Wrapper Frame - 240 400 Mpa

(D) Tensile Load - Acted During Swinging - 340 390 $\mathrm{Mpa}$

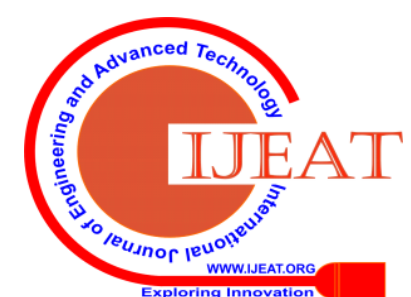


To Improve Internal RFT by Eliminating Wrapper Fouling with Bumper Issue in MF 9500 Models

\section{TABLE-VI: INVESTIGATION RESULTS AND DEFICIENCIES IDENTIFIED}

\begin{tabular}{|c|c|c|}
\hline $\begin{array}{l}\text { Contributing } \\
\text { conditions }\end{array}$ & $\begin{array}{l}\text { 4M correlations } \\
\text { (primary) }\end{array}$ & $4 \mathrm{M}$ correlations(Secondary) \\
\hline \multirow{4}{*}{$\begin{array}{l}\text { 1. RM Strength , } \\
\text { Welding Strength }\end{array}$} & \multirow{4}{*}{$\begin{array}{l}\text { 1-1.Wrapper } \\
\text { Frame mechanical } \\
\text { Properties }\end{array}$} & 1.1.1 RM - Not matching to the specification \\
\hline & & 1.1.2 Less Thickness of weld Channels \\
\hline & & 1.1.3 Welding Size, thickness less \\
\hline & & $\begin{array}{l}\text { 1.1.4 Wrapper Design - Not withstanding } \\
\text { Impact during swinging }\end{array}$ \\
\hline \multirow{3}{*}{$\begin{array}{l}\text { 2. Hood Swinging } \\
\text { Angle/ Distance }\end{array}$} & \multirow{3}{*}{$\begin{array}{l}2.1 \text { More swinging } \\
\text { angle / distance } \\
\text { creates excess load }\end{array}$} & 2.1.1 Wrapper stopper angle variation \\
\hline & & 2.1.2 Excess Swinging angle- Design \\
\hline & & $\begin{array}{l}\text { 2.1.3 Less Clearance between Wrapper \& } \\
\text { Bumper after swinging - Design }\end{array}$ \\
\hline
\end{tabular}

\begin{tabular}{|c|c|c|c|c|c|}
\hline $\begin{array}{l}\text { Location } \\
\text { check }\end{array}$ & Items to be checked & $\mid \begin{array}{c}\text { Measuremen } \\
\mathrm{t} \text { method }\end{array}$ & Tolerances & $\begin{array}{c}\text { Measured } \\
\text { value }\end{array}$ & Remarks \\
\hline \multirow{4}{*}{$\begin{array}{l}1-1 \\
\text {.Wrapper } \\
\text { Frame } \\
\text { mechanical } \\
\text { Properties }\end{array}$} & $\begin{array}{l}1.1 .1 \mathrm{RM} \text { - Not matching to the } \\
\text { specification }\end{array}$ & RM Testing & $\begin{array}{l}\text { Spec to IS1079- } \\
\text { Grade D } \\
240-400 \mathrm{MPA}\end{array}$ & $295 \mathrm{MPA}$ & OK \\
\hline & $\begin{array}{l}\text { 1.1.2 Less Thickness of weld } \\
\text { Channels }\end{array}$ & Vernier & $3 \pm 0.2 \mathrm{~mm}$ & $3 \mathrm{~mm}$ & OK \\
\hline & 1.1.3 Welding Size, thickness less & Metallurgy & $\begin{array}{l}3 \mathrm{~mm} \text { - Weld size } \\
\text { Spec CPS303 }\end{array}$ & $\begin{array}{l}3.5 \mathrm{~mm} \text {, as } \\
\text { per spec } \\
\text { CPS } 303\end{array}$ & ок \\
\hline & $\begin{array}{l}\text { 1.1.4 Wrapper Design - Not } \\
\text { withstanding Impact during swinging }\end{array}$ & FEA & - & $340-390 \mathrm{MPA}$ & Not OK \\
\hline \multirow{3}{*}{$\begin{array}{l}2.1 \text { More } \\
\text { swinging } \\
\text { angle / } \\
\text { distance } \\
\text { creates } \\
\text { excess load }\end{array}$} & $\begin{array}{l}2.1 .1 \text { Wrapper stopper angle } \\
\text { variation }\end{array}$ & Height Gauge & $90 \mathrm{Deg}$ & $90 \mathrm{Deg}$ & OK \\
\hline & 2.1.2 Excess Swinging angle- Design & $\begin{array}{c}\text { Bevel } \\
\text { Protractor }\end{array}$ & 55Deg & 55Deg & ок \\
\hline & $\begin{array}{l}\text { 2.1.3 Less Clearance between } \\
\text { Wrapper \& Bumper after swinging - } \\
\text { Design }\end{array}$ & Vernier & $20 \mathrm{~mm}$ & $20 \mathrm{~mm}$ & OK \\
\hline
\end{tabular}

\begin{tabular}{|c|c|c|}
\hline $\begin{array}{l}\text { Contributing } \\
\text { conditions }\end{array}$ & $\begin{array}{l}\text { 4M correlations } \\
\text { (primary) }\end{array}$ & $4 \mathrm{M}$ correlations(Secondary) \\
\hline \multirow{4}{*}{$\begin{array}{l}\text { 1. RM Strength, } \\
\text { Welding Strength }\end{array}$} & \multirow{4}{*}{$\begin{array}{l}\text { 1-1.Wrapper } \\
\text { Frame mechanical } \\
\text { Properties }\end{array}$} & 1.1.1 RM - Not matching to the specification \\
\hline & & 1.1.2 Less Thickness of weld Channels \\
\hline & & 1.1.3 Welding Size, thickness less \\
\hline & & $\begin{array}{l}\text { 1.1.4 Wrapper Design - Not withstanding } \\
\text { Impact during swinging }\end{array}$ \\
\hline \multirow{3}{*}{$\begin{array}{l}\text { 2. Hood Swinging } \\
\text { Angle/ Distance }\end{array}$} & \multirow{3}{*}{$\begin{array}{l}2.1 \text { More swinging } \\
\text { angle / distance } \\
\text { creates excess load }\end{array}$} & 2.1.1 Wrapper stopper angle variation \\
\hline & & 2.1.2 Excess Swinging angle- Design \\
\hline & & $\begin{array}{l}\text { 2.1.3 Less Clearance between Wrapper \& } \\
\text { Bumper after swinging - Design }\end{array}$ \\
\hline
\end{tabular}

Design Study - Impact Load

Impact Load $=1 / 2 \mathrm{M}^{*} \mathrm{~V}^{2}$ or $\mathrm{M}^{*} \mathrm{G}^{*} \mathrm{H}$

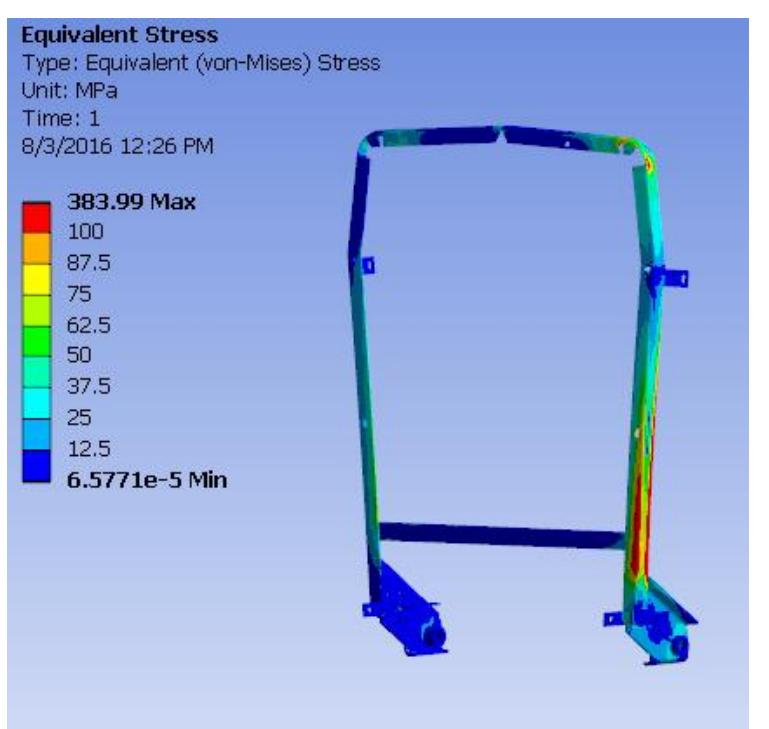

Fig. 10, 11. Impact Load Observed from 340- 390 MPA \& Leads to Deformation upto $9.95 \mathrm{~mm}$

Channel Strength as per IS1079 - Grade D - 240-400 MPA. Peak Load observed in Side channel area leading to bending.

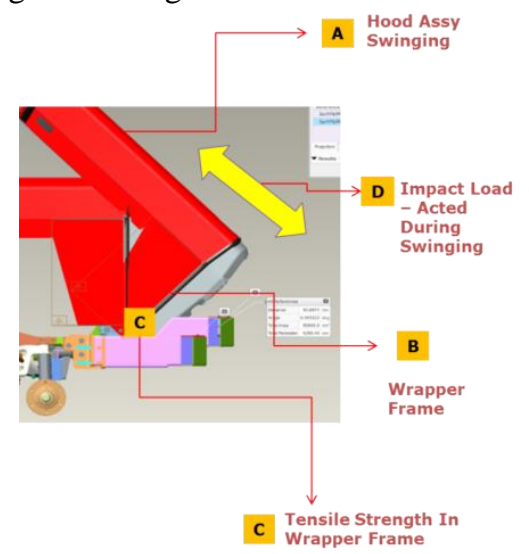

Fig. 12. Design Study - Swinging Study

Design gap between bumper and wrapper

Hood opening angle $55^{\circ}$ clearance between bumper and wrapper is 20 mm @ nominal

\section{STEP : 4 ERADICATE CAUSES}

Proposal -1

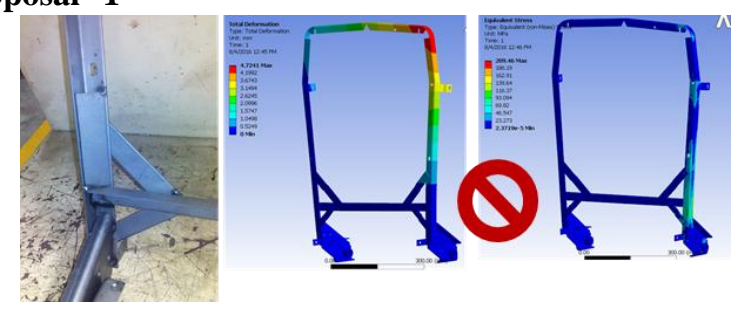

Fig. 13. Welding stiffener provision in Load acting area Impact Stress reduces but still deformation observed upto $5 \mathrm{~mm}$.

Published By: 
Trial Not Ok

Proposal -2 Swinging Angle - Modification

Present - Proposed

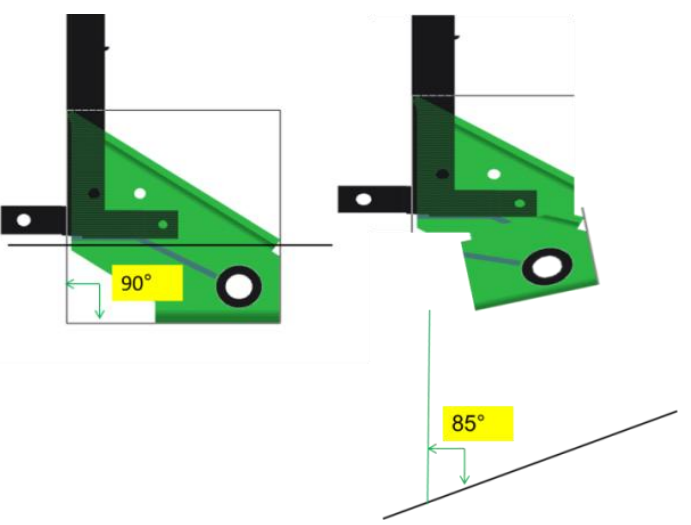

Fig. 14. Wrapper frame stopper plate angle $90^{\circ}$ to $85^{\circ}$

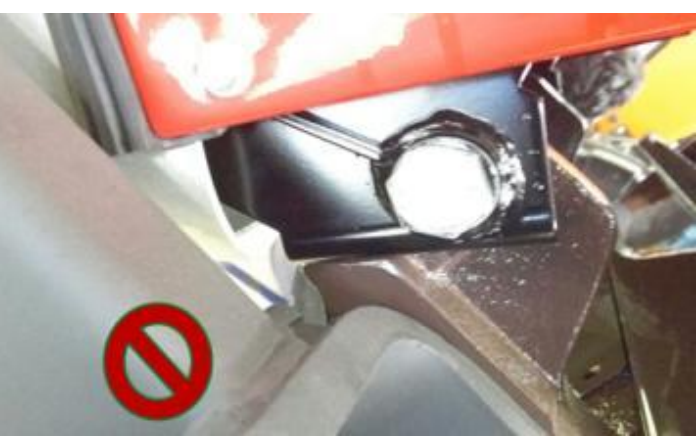

Fig. 15. Fitment Taken and still wrapper frame bent observed after swinging 10 Times

\section{Proposal -3}

As a Proposal adding the tubular section as shown in image. Which will reduce the Wrapper twist. Also It we will help to enhance the New hood lock Systems.

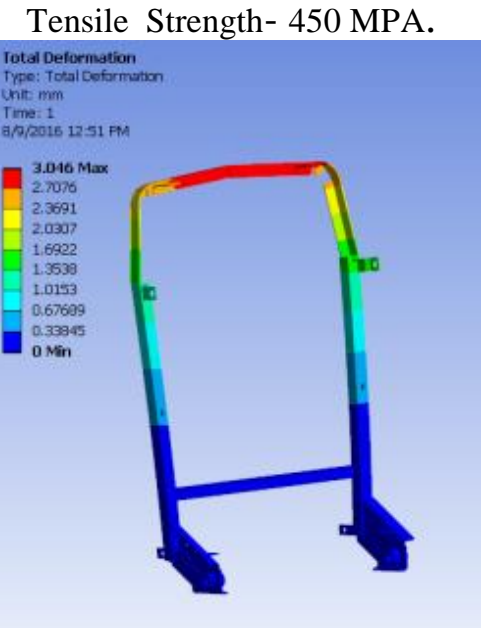

IS $4923: 1997$

\begin{tabular}{ccccccccc}
\multicolumn{7}{c}{ Table 1 Dimensions and Properties of Square Hollow Sections } \\
(Clause 8.1 and Note in Annex A)
\end{tabular}
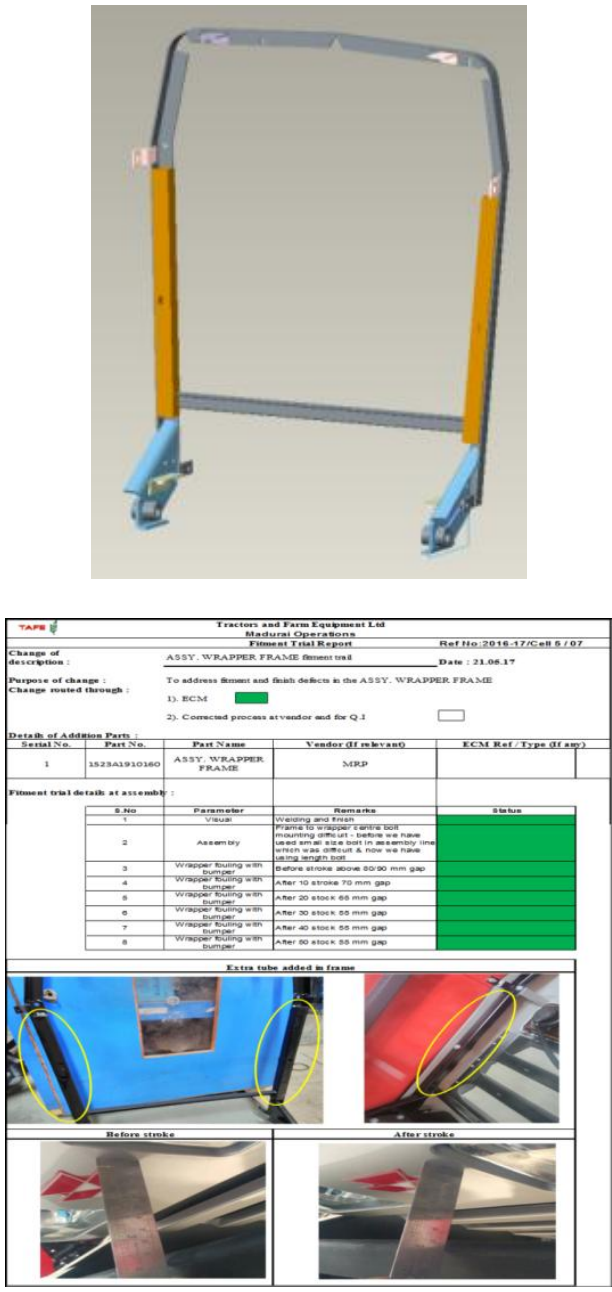

Fig. 16. Fitment Feedback found ok

\section{STEP : 5 ESTABLISH CONDITIONS}

TABLE-VII: REVISED QM MATRIX

\begin{tabular}{|c|c|c|c|c|c|}
\hline Process & Parameter & Specification & $\begin{array}{l}\text { Component } \\
\text { dimension }\end{array}$ & Torque & Compliance \\
\hline \multirow{4}{*}{$\begin{array}{l}\text { 1-1.Wrapper } \\
\text { Frame } \\
\text { mechanical } \\
\text { Properties }\end{array}$} & $\begin{array}{l}\text { 1.1.1 RM - Not matching } \\
\text { to the specification }\end{array}$ & \begin{tabular}{|c|} 
Spec to \\
IS4923-Grade \\
$25 * 25$ \\
450 MPA \\
\end{tabular} & $\oplus$ & $\otimes$ & $\oplus$ \\
\hline & $\begin{array}{l}\text { 1.1.2 Less Thickness of } \\
\text { weld Channels }\end{array}$ & $3 \pm 0.2 \mathrm{~mm}$ & $\oplus$ & $\otimes$ & $\oplus$ \\
\hline & $\begin{array}{l}\text { 1.1.3 Welding Size, } \\
\text { thickness less }\end{array}$ & $\begin{array}{l}3 \mathrm{~mm} \text { - Weld } \\
\text { size } \\
\text { Spec CPS303 }\end{array}$ & $\oplus$ & $\otimes$ & $\oplus$ \\
\hline & $\begin{array}{l}\text { 1.1.4 Wrapper Design - } \\
\text { Not withstanding Impact } \\
\text { during swinging }\end{array}$ & - & $\oplus$ & $\otimes$ & $\oplus$ \\
\hline \multirow{3}{*}{$\begin{array}{l}2.1 \text { More } \\
\text { swinging angle } \\
\text { / distance } \\
\text { creates excess } \\
\text { load }\end{array}$} & $\begin{array}{l}\text { 2.1.1 Wrapper stopper } \\
\text { angle variation }\end{array}$ & 90 Deg & $\oplus$ & $\otimes$ & $\oplus$ \\
\hline & $\begin{array}{l}2.1 .2 \text { Excess Swinging } \\
\text { angle- Design }\end{array}$ & 55Deg & $\oplus$ & $\otimes$ & $\oplus$ \\
\hline & $\begin{array}{l}2.1 .3 \text { Less Clearance } \\
\text { between Wrapper \& } \\
\text { Bumper after swinging - } \\
\text { Design }\end{array}$ & $20 \mathrm{~mm}$ & $\oplus$ & $\otimes$ & $\oplus$ \\
\hline
\end{tabular}

STEP : 6 IMPROVE CONDITIONS

Cost Impact- Calculation

Part No: 1523A93501

Part Name: Wrapper Frame

Cost Impact: 72 Rs/ Wrapper Frame

Wrapper Cost: 1642/ Piece

Avg Rejections: 16 nos month,

Rejection Cost: Rs 26272

Avg Prod Volume: 60nos /

Month (748 model) 
Cost Increase due to Improvement: $72 * 60=4320$

Cost Saving: (Direct) 21952/Month

\section{STEP : 7 MAINTAIN CONDITIONS}

Perform Checks ( Horizontal Deployment)

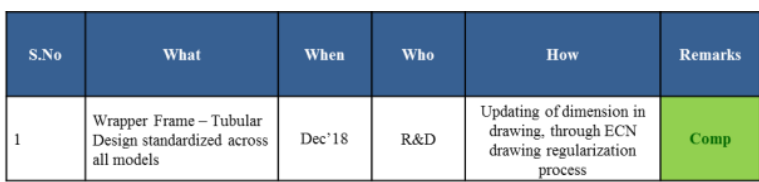

\section{RESULT}

\section{STEP : 7 MAINTAIN CONDITIONS}

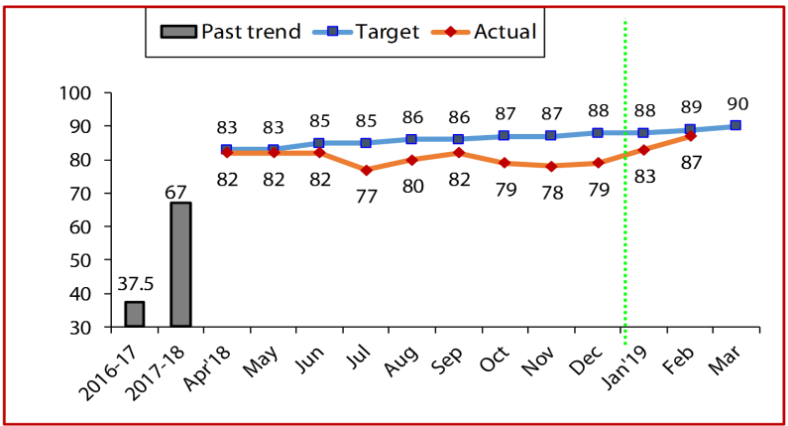

Fig. 17. Maintain Conditions

\section{CONCLUSION}

Proposed Models are capable increasing the first pass yield from $83 \%$ to $89 \%$. It is also noted that warranty cost due to wrapper damage is significantly reduced and rework for the proposed model capable of reducing the cost by Rs 22000 per month. The proposed model is also capable of reducing the sick effect considerably. The maximum delivery time for the existing model is 3 and proposed model is capable of delivering the items within one day. Aesthetic model of the proposed reduces the inspector fatigue and avoids the wrapper damage.

\section{REFERENCES}

1. D.R .Kiran (2018) "Total productive maintenance article"

2. Luis A.Nolasco (2012) "Angular momentum during 90-degree turns"

3. R. AjitShenoib (2018) "Experimental and numerical study on ultimate strength of steel tubular members"

4. Yazıcı Hasan, Şahan Arel VolkanTabak (2012)“The effects of impact loading on the mechanical properties"

5. D.Kumar RajdeepSarkarK. SivaKumar (2018) "Effect of Tensile strength of steel frame"

6. Jonathan D.Glassman (2011) "Effectiveness of welded stiffeners"

7. Kim Rasmussen (2009) "System reliability-based Direct Design Method for frames steel hollow sections"

8. Michael C.Granatosky (2019) “The mechanical origins of arm-swinging"

9. Satyanarayan Gangaram Pitroda (1997) “IS 4923: Hollow steel sections for structural use - Specification"

10. Hyosung Polyketone (2015) "Engineering plastic has high strength and excellent wear resistance"

11. JiadingWang (2018) “Changes in tensile strength and microstructure due to vibration"

12. I.A.Yakimo ,S.S.Voronin, E.A.Maklakova, A.S.Maklakov (2017) "Analysis of the Impact Load Causes"

13. XiaoyiLana JunboChen (2018) “The continuous strength method for the design of high strength steel tubular sections"
14. Paul A.Tres (2017) "Strength of Material for Plastics"

15. Mr.Ranjeet,M.Jadhav,Mr.MorosinAlessandro, Prof.S.H.Sawant (2015) "Total Productive Maintenance Theoretical Aspect"

16. Professor Paolo Carbone (2011) “Measurement \& Instrumenation”

\section{AUTHORS PROFILE}

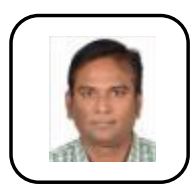

Dr. S. Rajesh completed his B. E in Mechanica Engineering and M.E. ( CAD) from Alagappa Chettiar Government College of Engineering and Technology, Karaikudi, Ph.D (Machining of Metal of Matrix Composites) from Kalasalingam Academy of Research and Education, Krishnankoil in the year 2014, and thesis highly recommended by both the examiners. In the year 2018, I have received DST - AMT project for the worth of Rs. 25,72,400 and completed one IEDC project for the worth of Rs. 1,00,000. He has published more than 25 Journal papers (Impact Factor and Scopus Indexed) and more than 40 International Conference papers. 